\title{
Balkanologie
}

Balkanologie Revue d'études pluridisciplinaires

Vol. IX, n' 1-2 | 2005

Volume IX Numéro 1-2

\section{Du réveil national au « réveil » religieux ?}

Le cas de la Serbie au tournant du XXI ${ }^{\mathrm{e}}$ siècle

From National Awakening to Religious "Awakening"? The case of Serbia at the turning of 21st century

\section{Yves Tomić}

\section{OpenEdition \\ Journals}

Édition électronique

URL : http://journals.openedition.org/balkanologie/594

DOI : 10.4000/balkanologie.594

ISSN : 1965-0582

\section{Éditeur}

Association française d'études sur les Balkans (Afebalk)

\section{Édition imprimée}

Date de publication : 1 décembre 2005

ISSN : $1279-7952$

\section{Référence électronique}

Yves Tomić, «Du réveil national au « réveil » religieux? », Balkanologie [En ligne], Vol. IX, n 1-2 | 2005, mis en ligne le 13 janvier 2010, consulté le 17 décembre 2020. URL : http://journals.openedition.org/ balkanologie/594 ; DOI : https://doi.org/10.4000/balkanologie.594 


\title{
DU RÉVEIL NATIONAL AU « RÉVEIL » RELIGIEUX? LE CAS DE LA SERBIE AU TOURNANT DU XXI İ̀me SIÈCLE
}

\author{
Yves Tomic*
}

L'historiographie serbe accorde un rôle important à l'Église orthodoxe dans la préservation et la construction de l'identité nationale serbe. Son rôle est surtout souligné pour la période allant de la disparition de l'État médievval serbe en 1459 aux insurrections libératrices du début du XIX ${ }^{\text {ème }}$ siècle (18041813 et 1815). Néanmoins, son rôle a été moins important dans la définition de la nation serbe contemporaine établie au XIX ${ }^{\text {ème }}$ siècle comme une nation multiconfessionnelle orthodoxe, catholique et musulmane ${ }^{1}$. L'Église serbe orthodoxe n'en pas moins été un acteur institutionnel et social important au sein de l'État de Serbie puis de Yougoslavie, entre 1918 et 1941.

$\mathrm{Au}$ cours de la période communiste, l'Église serbe orthodoxe se retrouve marginalisée. Elle réapparait sur la scène sociale de manière significative au cours des années 1980 . Il s'agira de préciser le contexte dans lequel l'Église serbe orthodoxe s'est imposée comme acteur social de poids. Quel a été son rôle dans le mouvement national serbe de la seconde moitié des années 1980 ? Comment s'est-elle positionnée pendant les guerres de dissolution yougoslave au cours des années 1990 ? Si l'Église serbe orthodoxe s'est imposée comme l'une des premières institutions sociales de référence au cours des années 1990 , a-t-elle réussi à renforcer la religiosité des citoyens serbes? Peut-on parler d'un retour du religieux?

Cet article analyse l'évolution de l'Église serbe orthodoxe à partir de ses courants dominants. L'auteur a, bien entendu, conscience qu'elle représente une organisation plus diversifiée et complexe qu'on ne le pense habituellement.

\footnotetext{
'Ingénieur d'études (BDIC, Université Paris X.Nanterre), chercheur associé au LASP (CNRS, Université Paris X-Nanterre). E-mail : yves.tomic@bdic.fr

${ }^{1}$ Selon les conceptions de Vuk Karadżić, reprises ensuite par une partie importante de l'élite intellectuelle serbe. Tomić (Yves), La Serbie du prince Milośa à Milošević, Bruxelles : P.I.E. Peter Lang, 2003, PP. 44-48.
} 


\section{LA POSITION DE L'ÉGLISE SERBE ORTHODOXE DANS LA YOUGOSLAVIE COMMUNISTE}

Comme l'ont souligné l'historienne Radmila Radić et le sociologue de la religion Dragoljub B. Djordjević, peu d'études systématiques ont été consacrées à l'Église serbe orthodoxe pendant la période communiste. Ce n'est qu'au début des années 1990 qu'un intérêt pour cette institution s'est manifesté, et surtout de la part de sociologues de la religion ${ }^{2}$.

En 1946, la nouvelle Constitution yougoslave proclame le principe de séparation de l'Église et de l'État tout en reconnaissant le droit de pratiquer la religion. L'Église serbe orthodoxe se retrouve socialement marginalisée. Elle ne peut plus s'occuper d'éducation et les mariages religieux sont supprimés. La réforme agraire l'ampute d'une grande partie de ses terres3. En 1958, elle perd également une part considérable de son patrimoine immobilier et foncier. Globalement, c'est la période de 1945 à 1955 qui est la plus difficile pour l'Église serbe. Même si les relations hiérarchiques entre l'État et l'Église s'améliorent au début des années 1950, à l'échelle locale, les tracasseries perdurent ${ }^{4}$. Sa presse demeure également limitée5. L'Église parvient toutefois à restaurer de nombreux monastères, églises et chapelles.

Outre son influence sociale diminuée, elle subit également un rétrécissement des diocèses sous sa juridiction : elle perd ceux de Tchécoslovaquie entre 1945 et 1948 et, en 1969, une partie de ceux de Roumanie sont rattachés à l'Église orthodoxe roumaine. La perte la plus importante est celle des diocèses de la République de Macédoine qui se constituent, en 1967, en Église orthodoxe autocéphale de Macédoine sans l'accord du Patriarcat serbe. De surcroît, les autorités communistes encouragent l'autonomisation des diocèses du Monténégro sans pour autant qu'une Église indépendante ne soit (re)constituée.

L'Église serbe orthodoxe entretient une relation particulière à la nation comme d'ailleurs l'ensemble des Églises orthodoxes. Certaines de ces Églises sont manipulées et utilisées dans les régimes communistes d'Europe orientale en raison de leur nationalisme, notamment en Roumanie et en Bulgarie. Étant donné l'hétérogénéité nationale de l'État yougoslave, l'Église serbe orthodoxe n'a jamais été encouragée dans son expression nationaliste. Cela ne l'empêche pas de prendre à certains moments des positions s'en rapprochant : lors de l'essor du mouvement national croate entre 1967 et 1971 ; face aux tentatives d'au-

\footnotetext{
${ }^{2}$ Radić (Radmila), Verom protiv vere (Avec foi contre la foi), Beograd : INIS, 1995, p. 9.

3 Sur les 173367 hectares de terres confisqués par l'État, 70000 appartenaient à l'Église serbe orthodoxe.

4 Alexander (Stella), Church and State in Yugoslavia since 1945, Cambridge : Cambridge University Press, 1979, p. 251.

5 Voir le chapitre sur l'Église orthodoxe serbe dans l'ouvrage de Ramet (Pedro), ed., Religion and Nationalism in Soviet and East European Politics, Durham : Duke University Press, 1984, pp. 154-155.
} 
tonomisation de l'Église orthodoxe au Monténégro; et lors de la création de l'Église autocéphale de Macédoine. Les Monténégrins et les Macédoniens sont considérés comme des membres de la nation serbe : les Macédoniens étant dénommés "Serbes du Sud ». Sous la période titiste, l'Église serbe orthodoxe se présente comme une institution nationale et la forteresse de l'identité nationale, notamment lorsqu'elle installe les reliques de l'empereur Dušan à l'église Saint-Marc de Belgrade en 1968. Certains ecclésiastiques sont d'ailleurs condamnés à plusieurs années de prison pour avoir fait preuve de nationalisme lors de leurs prêches ${ }^{6}$. Toutefois, la perception de la nation n'est pas unique et certains théologiens orthodoxes mettent en avant les valeurs universelles du christianisme ${ }^{7}$. Du fait de l'imbrication du national et du religieux, il n'est pas étonnant de voir l'Église orthodoxe se préoccuper des intérêts nationaux au cours des années 1980 .

\section{LA REVITALISATION DE L'ÉGLISE DANS LES ANNÉES 1980}

La revitalisation de l'Église serbe orthodoxe est favorisée par le contexte de crise économique, sociale et idéologique des années 1980 . Les valeurs que portait l'Église jusqu'en 1945 ont été rejetées alors qu'elles étaient porteuses de sens et d'enracinement dans la société pour les citoyens serbes (traditions, morale patriarcale, valeurs religieuses et histoire nationale $)^{8}$. Lors du dépérissement progressif du régime communiste, on constate un rapprochement de certains individus vers l'Église serbe orthodoxe, vecteur des valeurs et symboles nationaux.

La plupart des enquêtes réalisées au cours des années 1980 concluent néanmoins à une forte sécularisation parmi les habitants serbes des territoires de tradition orthodoxe 9 . Le nombre des croyants et pratiquants orthodoxes chute considérablement sous le régime communiste : dans une enquête menée dans la région de Niš en 1982, 23,8 \% des personnes interrogées se déclarent croyantes - contre, à la même époque, 50,5\% en Slovénie (1981-1982) et $44,6 \%$ dans la région de Zagreb - et une seconde, réalisée en 1984 à Belgrade,

6 L'évêque Vasilije de Ziča en 1972, Sava Branković en 1973, Nedjo Janjić en 1980. Voir Ramet (Pedro). "Religion and Nationalism in Yugoslavia ", in Ramet (Pedro), op. cit., p. 162.

7 Radić (Radmila), "Srpska pravoslavna crkva u poratrim i ratnim godinama : 1980-1995 " (I'Église orthodoxe serbe dans les années d'avant-guerre et de guerre), Ogledi (suppl. de Republika, (121-122), o131/08/95\}, p. II.

8 D'après le sociologue Blagojević (Mirko), "Vezanost ljudi za religiju i crkvu na pravoslavno homogenim prostorima " (Le rapport des gens à la religion et à l'Église dans les territoires orthodoxes homogènes) in Religija rat-mir (Religion-guerre-paix), Niš, 1994, p. 209.

9 Le processus de sécularisation affecta moins les catholiques et les musulmans. Ibid., p. 207. 
en indique $10 \%^{10}$. Or, la puissance d'une Église dépend essentiellement du nombre de croyants déclarés et de pratiquants se rendant régulièrement à l'église. $\mathrm{Au}$ cours des années 1980, et cela sera constaté aussi ultérieurement, les personnes se déclarant de confession orthodoxe et étant liées traditionnellement à la religion (respect de certaines pratiques comme le baptême et l'enterrement religieux, célébration des fêtes religieuses, etc.) ne remplissent pas leurs obligations religieuses auprès de l'Église. Trois facteurs ont été avancés pour expliquer cette forte sécularisation : l'affaiblissement et la souffrance de l'Église serbe orthodoxe au cours de la Seconde Guerre mondiale, le poids du régime communiste (surtout à travers l'idéologisation de la vie sociale et de l'éducation et l'enseignement athée) et les faiblesses internes de l'Église ${ }^{11}$. En outre, les connaissances de la population, et des croyants en particulier, sur les principes du christianisme et de l'orthodoxie ne sont pas importantes. La décomposition de l'idéologie communiste et l'engagement de l'Église dans la défense de la cause nationale permettent une revitalisation des pratiques religieuses, surtout dans la seconde moitié des années 1980 et plus précisément à la fin de cette décennie. Mais force est de constater que l'Église orthodoxe ne constitue pas une institution puissante et influente au cours de la période communiste.

L'Église serbe orthodoxe saisit les événements du Kosovo en 1981 pour revenir sur la scène publique et sortir de son isolement. Au cours des années 1980, l'Église orthodoxe se fait de plus en plus présente. En 1984, elle obtient l'autorisation de construire un grand édifice (église Saint Sava) à Belgrade, ainsi que de nouveaux locaux pour la faculté de théologie ${ }^{12}$. En fait, il s'agit de la poursuite de la construction d'un grand temple qui avait été interdite après 1945. La hiérarchie de l'Église avait plusieurs fois demandé la reprise de la construction, mais ce n'est que sous la présidence de Dušan Čkrebić (1984-1986) que l'autorisation est accordée (juin 1985) ${ }^{13}$. La construction de la faculté de théologie est réalisée avec l'aide et sous le contrôle de l'État. La faculté est appelée à renforcer la dimension intellectuelle de l'Église serbe orthodoxe. Les rassemblements religieux se multiplient, les ecclésiastiques accordent des entretiens dans les médias, de nouvelles revues ecclésiastiques sont créées (Glas crkve fin 1984-début 1985 en particulier). Le discours des ecclésiastiques passe progressivement de la question de la construction et de la rénovation des églises à celle de la position du peuple serbe dans la Yougoslavie socialiste.

10 Djordjević (Dragoljub B.), Djurović (Bogdan), " Sekularizacija i pravoslavlje : slučaj Srba " (Sécularisation et orthodoxie : le cas des Serbes), in Djordjević (Dragoljub B.), ed., Povratak svetog ? Zbornik tekstova (Le retour du sacré ? Recueil de textes), Niš : Gradina, 1994, p. 222.

${ }^{11}$ Ibid., p. 221.

12 À propos de la construction de l'église Saint-Sava, voir Aleksov (Bojan), " Nationalism in Construction : The Memorial Church of St. Sava on Vracar Hill in Belgrade ", Balkanologie, 7(2), 2003.

13 Čkrebić (Dušan), Zapis na pesku : sećanja (Note sur le sable : mémoires), Beograd : Prosveta, 1995, p. 141. 


\section{LE COMBAT NATIONAL}

Ce changement d'orientation sous le système communiste est impulsé par un courant d'ecclésiastiques plus jeunes (Anatasije Jevtić, Amfilohije Radović, Irinej Bulović, Artemije Radosavljević). Ce groupe de religieux appartient à la même génération et a pour pères spirituels Justin Popović et Nikolaj Velimirović, théologiens de l'entre-deux-guerres. Le premier, évêque de l'avantguerre, avait été écarté et isolé après 1945, le second avait dû quitter la Yougoslavie pour les États-Unis car il était considéré comme un " traitre " et un " nationaliste clérical " par les nouvelles autorités communistes. Leurs écrits, mis en avant par le groupe de religieux, constituent les fondements idéologiques du combat de l'Église pour la cause nationale. Justin Popović et Nikolaj Velimirović se sont opposés en leur temps à l'influence du monde occidental dans la société yougoslave de l'époque. Selon Nikolaj Velimirović, la Seconde Guerre mondiale a été la conséquence de la sécularisation de l'Europe infidèle et peut s'expliquer par la trahison de Dieu et des valeurs chrétiennes ${ }^{14}$. Il s'est également distingué par ses écrits antisémites dans lesquels il estimait que les souffrances des juifs pendant la guerre ont constitué la vengeance de Dieu pour la mort du Christ ${ }^{15}$. Ces ecclésiastiques plus jeunes contestent l'autorité du patriarche German, depuis 30 ans à la tête de l'Église serbe orthodoxe. Ils lui reprochent entre autres son manque de combativité contre le régime communiste et l'athéisme.

La situation de tension au Kosovo est l'élément moteur du dynamisme de l'Église. Le groupe de religieux se manifeste pour la première fois en février 1982 , soulevant la question nationale au sein de l'Église dans une lettre ouverte au patriarche German et au synode des évêques de l'Église serbe orthodoxe. Ils y soulignent entre autres la situation difficile du clergé, ainsi que des croyants dans la province du Kosovo, et reprochent à la hiérarchie de l'Église, et à son organe Pravoslavlje, de n'être pas suffisamment sensibles à ce genre de problèmes : " La presse de l'Église ne donne aucune information sur les pogroms, sur les exactions commises contre le clergé et les fidèles orthodoxes au Kosovo. Elle

\footnotetext{
14 Bajford (Jovan), " Mučeništvo u Dahauu kao "zamenjeni mit" " (Le martyr à Dachau comme mythe de substitution), Danas, 06/04/05, p. 31. Au cours de la première moitié du XXeme siècle, Nikolaj Velimirović, évêque d'Ohrid et Ziča, était l'un des ecclésiastiques les plus respectés. Il se prononçait pour l'établissement d'une société basée sur les valeurs chrétiennes orthodoxes, nationalistes et monarchistes. Il rejetait fermement les traditions et valeurs occidentales telles que l'individualisme, l'égalité, la tolérance religieuse, la démocratie. Bajford (Jovan), " Glavni glas hrišćanskog nacionalizma u Srbiji " (La principale voix du nationalisme chrétien en Serbie), Danas, 05/04/05, p. 24.

15 Nikolaj Velimirović a écrit ses textes les plus antisémites alors qu'il était paradoxalement interné au camp de Dachau en 1944. Ses notes de Dachau ont été publiées pour la première fois en 1985 en Allemagne avant de l'être en Serbie sous le titre de Reč srpskom narodu kroz tamnički prozor (Parole au peuple serbe à travers la fenêtre de la prison).
} 
ne donne aucune information sur les destructions, les incendies et les actes blasphématoires commis contre les sanctuaires du Kosovo ${ }^{16}$.

Les auteurs de cette lettre ouverte, exerçant dans l'éparchie de Raška et de Prizren, demandent à l'Église de reconsidérer sa place dans la société et ses relations avec le pouvoir, et l'appellent à sortir de son silence. En raison des graves critiques portées à l'encontre de la hiérarchie de l'Église, leur lettre n'est pas publiée dans la presse ecclésiastique serbe en Yougoslavie mais à l'étranger. Le journal du patriarcat serbe Pravoslavlje fait allusion aux critiques qui circulent dans les rangs de l'Église en reprenant un texte de l'organisation du clergé orthodoxe de Yougoslavie (créée à l'initiative du régime après 1945), publié dans son organe Vesnik. À propos du silence de l'Église orthodoxe face à la " situation de terreur " au Kosovo, ces religieux écrivent :

L'Église ne se tait pas : elle fait ce qu'elle peut, consciente de la gravité du moment. Elle ne souhaite pas " l'escalade du nationalisme serbe " et elle se comporte dans une grande mesure de façon constructive : elle parle là où cela a un sens, appelant à la conscience, aux intérêts étatiques, sociaux et nationaux. C'est exact, l'Église prône la tolérance - et elle continuera à le faire, car il s'agit du commandement du Christ. De cette façon, elle éduque et renforce son peuple afin qu'il puisse résister à chaque épreuve, car « Dieu est fidèle i il ne permettra pas que vous soyez tentés au-delà de vos forces. Avec la tentation, il vous donnera le moyen d'en sortir et la force de la supporter " (1 Cor. 10, 13). L'Église n'appelle pas à la vengeance mais parle de pardon; elle ne demande pas l'application de la force mais appelle la conscience ${ }^{17}$.

En avril 1982, un groupe de 21 popes (Atanasije Jevtić, Irinej Bulović, Amfilohije Radović, etc.), dans la ligne de la lettre ouverte du 26 février 1982, rédige un appel d'une portée plus générale à l'adresse des autorités fédérales, serbes, ainsi qu'au synode de l'Église. Ces popes réagissent à la situation des Serbes du Kosovo afin de protéger cette région considérée comme "l'être biologique et spirituel du peuple serbe ". Leur appel est publié dans le journal Pravoslavlje en date du 15 mai $1982^{18}$. Ce texte est le point de départ de l'investissement de l'Église orthodoxe, sinon d'une partie de ses rangs, dans la défense des intérêts nationaux serbes. Par leur appel adressé aux autorités, ces ecclésiastiques se placent dans les champs politique et public. Leur vision de la

16 Cette lettre est reprise dans l'ouvrage de Jevtić (Atanasije), Dossier Kosovo, Lausanne : L'Age d'Homme, 1991, pp. 149-151.

17 Pravoslavlje, $15 / 02 / 82$, p. 2. En outre, on peut signaler que Pravoslavlje reprit dans son numéro du 15/01/82 un texte sur les violences contre les Serbes du Kosovo publié en décembre 1981 dans le périodique serbe Duga.

18 Pravoslavije, 15/05/82, pp. 1-4. 
nation serbe, inscrite dans le champ religieux, est inséparable de la région du Kosovo considérée comme le principal lieu de mémoire des Serbes :

Une chose doit être claire pour tous : qu'il s'agisse du passé, du présent ou de l'avenir, le peuple serbe n'a pas de mot plus cher que le norn de Kosovo, ni de réalité plus précieuse, ni de sanctuaire plus grand. Pour les Serbes, la question du Kosovo ne se réduit pas simplement à une question démographique, ni à une question de province, de région autonome, ou de république. Il s'agit de quelque chose d'infiniment plus grand et d'infiniment plus élevé. (...)

La question du Kosovo est une question d'identité spirituelle, culturelle et historique du peuple serbe. Depuis l'oeuvre de Saint Sava, c'est dans la réalitë du Kosovo que le peuple tout entier a trouvé, pour la première fois, l'expression de son unité et de son intégrité. ${ }^{19}$

Les auteurs de l'appel comparent le destin du peuple serbe à celui des Juifs qui, " après 2000 ans de souffrance et à l'encontre de toute logique historique " étaient retournés à Jérusalem. Ils replacent les exactions (menaces de mort, pillages, profanations de tombes, violences exercées sur les moines et les écoliers, etc.) dont sont victimes les Serbes, dans le contexte historique de la région. Selon eux, la situation de ces derniers au début des années 1980 est identique à celle qu'ils ont connue sous l'Empire ottoman et plus particulièrement à celle de la fin du XIX ${ }^{\text {ème }}$ siècle où ils ont subi d'importantes violences. En outre, ils mettent en avant l'exode forcé des Serbes et considèrent ce phénomène comme un génocide : "Sans exagération aucune, on peut dire qu'au Kosovo, le peuple serbe subit un lent génocide bien planifié. Car s'il n'en était rien, que signifierait alors cette thèse "d'un Kosovo ethniquement pur", défendue et mise en pratique sans honte par ceux qui provoquent des expulsions ininterrompues? 220 .

L'appel est donc une réaction aux exactions commises à l'encontre des Serbes du Kosovo ainsi qu'à leur " exode forcé " de cette province. Il apparait clairement dans ce document que ces ecclésiastiques ont pris conscience du déclin serbe au Kosovo qui est devenu progressivement, du point de vue démographique, une région albanaise. C'est ce déclin qui les motive à agir afin de préserver le caractère serbe du Kosovo. En fait, l'Église y défend aussi ses propres intérêts et son riche patrimoine. Afin de réveiller et de gagner l'opinion à sa cause, ainsi que de provoquer un retournement de situation, elle emploie des mots à fortes charges émotionnelles, tels que celui de génocide, qui, en fait, ne correspond pas à la réalité. 
La question du Kosovo est abordée lors du synode annuel de l'Église qui se déroule entre les 14 et 21 mai 1982. Les responsables de l'Église se défendent de se taire sur les exactions commises contre la population serbe du Kosovo et rappellent qu'ils ont en tout temps transmis les plaintes des ecclésiastiques auprès des autorités compétentes. Le synode décide de rédiger un rapport sur les violences commises contre les Serbes et, plus précisément, contre les religieux depuis l'année 1968 et sur les suites données par l'Église auprès des autorités politiques : ce document sera publié dans le journal du Patriarcat Pravoslavlje le 15 novembre 1982.

À partir de 1982, le thème du Kosovo ne quitte plus la presse orthodoxe et Atanasije Jevtić s'impose comme le principal acteur idéologique de cet engagement de l'Église pour le Kosovo. En juin 1982, il aborde la question du Kosovo et évoque la volonté des Albanais d'exterminer les Serbes de cette province ${ }^{21}$. Il revient également sur l'appel des 21 et les critiques publiées dans la presse en Serbie et en Croatie en se concentrant sur les remarques de journalistes de Croatie (Nenad Ivanković, Zorajda Runić). Un article sur l'exode des Serbes, à travers la poésie patriotique d'Aleksa Šantić, affirme que les Serbes quittent le Kosovo ainsi que d'autres Républiques ${ }^{22}$. Selon l'auteur, ce problème doit affecter l'ensemble de la communauté yougoslave dans la mesure où les Serbes constituent une force de cohésion pour l'État yougoslave. Les attaques contre les Serbes sont ainsi considérées comme des attaques contre la Yougoslavie. Le chauvinisme albanais est inscrit dans la continuité de la politique antiserbe d'Ante Pavelić pendant la Seconde Guerre mondiale. En outre, afin de défendre la cause des Serbes du Kosovo à l'étranger, un groupe d'évêques se rend aux États-Unis auprès du Congrès et du Département d'État.

A partir de la fin 1983, Atanasije Jevtić publie plusieurs textes sur le Kosovo tout en les élargissant aux crimes perpétrés par les Ustaše croates au cours de la Seconde Guerre mondiale. Il évoque les viols contre les femmes serbes au Kosovo, les pressions albanaises contre les religieux orthodoxes. Son attention se porte uniquement sur les violences, les exactions commises contre des Serbes. Il consacre ses recherches à cette thématique et le produit, hormis ses articles parus dans la presse, en est un ouvrage, véritable chronique des souffrances des Serbes du Kosovo et de la Métochie entre 1941 et $1990^{23}$. Il aborde, par la suite, la question des camps où ont été détenus et exterminés des Serbes entre 1941 et 1945, ainsi que celle des fosses communes où ils ont été en-

21 Pravoslavlje, (366), 15/06/82.

22 Jević (Hrizostom), * Iseljavanje Srba u Svetlosti rodoljubivih pesama Alekse Šantića " (L'exode des Serbes à la lưrnière des poèmes patriotiques d'Aleksa Šantić), Pravoslavlje, 15/12/82, p. 6 .

23 Jevtic (Atanasije), Stradanja Srba na Kosovu i Metohiji od 1941, do 1990 (Les souffrances des Serbes au Kosovo et en Métochie de 1941 à 1990), Priština : Jedinstvo, 1990. 
terrés ${ }^{24}$. Les textes en question soulignent l'identité de martyr de l'Église et du peuple serbes développée par cette institution. Atanasije Jevtić y lie le génocide commis par les Ustaše aux événements du Kosovo ${ }^{25}$. Dans cette même orientation, l'organe du Patriarcat publie également des textes, sur la base d'archives, au sujet des crimes et des violences exercés à l'encontre des Serbes par les Albanais dans la seconde moitié du XIXème siècle dans l'Ancienne Serbie (Kosovo). À partir de 1984, le thème du génocide contre les Serbes de Croatie au sein de l'État indépendant croate occupe une place de plus en plus importante dans la presse ecclésiastique, et plus particulièrement dans la seconde moitié des années 1980 .

Au cours de cette période, étant donné la nouvelle politique des autorités communistes en Serbie à partir de 1987 , l'Église peut s'exprimer plus librement et joue un rôle non négligeable dans le grand mouvement de réveil des sentiments nationaux. Lors de son synode annuel régulier qui se déroule du 23 mai au 2 juin 1987 à Peć au Kosovo puis à Belgrade, l'Église porte une attention particulière à "l'exode tragique et au génocide contre le peuple serbe au Kosovo " ainsi que dans certaines parties de la Serbie du sud-est (éparchie de Vranje) ${ }^{26}$. Pour la première fois, la direction de l'Église utilise le terme de génocide dans un document officiel. La rédaction de Glas Crkve, à la pointe du combat national de l'Église, insiste sur la nécessité de formuler un programme de travail sur le Kosovo avant la tenue du synode. Elle propose la création d'un comité pour le Kosovo, chargé de suivre la vie religieuse et de réagir aux menaces visant l'Église, et d'un bulletin d'information du Patriarcat consacré à cette question. Le synode s'oppose aux exigences de Glas Crkve mais n'en manifeste pas moins son inquiétude quant à la situation dans la province du sud de la Serbie. En 1987. l'Église prépare l'organisation du $60 o^{\text {ème }}$ anniversaire de la bataille du Kosovo qui doit avoir lieu en juin 1989. La même année, elle accorde une place significative à la commémoration de la personnalité de vuk Karadžić à l'occasion du bicentenaire de sa naissance. Elle saisit cette occasion pour défendre l'emploi de l'alphabet cyrillique et protester contre sa faible utilisation dans l'administration et les médias en Serbie ${ }^{27}$.

24 Ces textes furent publiés dans les numéros suivants de Pravoslavlje : (400) du 15/11/83, (404) du 15/01/84 et $(405)$ du $01 / 02 / 84$. Atanasije Jevtić les publia à comptes d'auteur en 1984 sous le titre de : Jevtlé (Atanasije), Od Kosova do Jadovna : putni zapisi jeromonaha Atanasija Jevtica (Du Kosovo à Jadovno : carnet de voyage du moine Atanasije Jevticj), Beograd : A. Jevtic, 1984.

25 " Immédiatement après la création de leur État Indépendant Croate malfamé, les oustachis ont commencé le soi-disant "nettoyage du terrain", c'est-à-dire qu'ils ont essayé de créer le plus rapidement possible un "espace croate pur" raciste, quelque chose de semblable à l'aspiration actuelle à un "Kosovo ethniquement pur" ». Ibid, p. 30.

26 Pravoslavlje, 01/06/87, p. 7 .

27 Terzić (Dragan), "O Slavljenju Vukove godine "(A propos de la célbration de l'année de Vuk), Pravoslavlje, 15/05/87, p. 2. 
En 1988, l'Église organise une action fortement symbolique et révélatrice de sa volonté d'affirmer sa présence dans la société serbe. Elle entreprend de transférer les reliques de Saint Lazar, le fameux prince ayant péri lors de la bataille du Kosovo, au monastère de Ravanica où celui-ci avait décidé de reposer. La décision avait déjà été prise en 1954 ; elle est appliquée pour marquer le $600^{\text {ème }}$ anniversaire de son " martyre ${ }^{28}$. Ses reliques partent de Belgrade pour le monastère de Vrdnik dans la Fruška Gora (Voïvodine) le 28 juin 1988, jour de la Saint Guy (Vidovdan), avant d'être transportées à travers les territoires serbes de Bosnie-Herzégovine et de Serbie pour le monastère de Gračanica où doit se dérouler la célébration du $600^{\text {ème }}$ anniversaire de la bataille du Kosovo en juin $1989^{29}$. Les reliques arrivent le 3 septembre 1988 au monastère d'Ozren dans l'évêché de Tuzla et de Zvornik où plus de 50000 personnes assistent aux cérémonies organisées à cette occasion ${ }^{30}$. Les reliques sont ensuite conduites au monastère de Ravanica.

Plus la crise du communisme yougoslave s'approfondit, plus l'Église se présente comme une institution nationale dont la principale tâche est de préserver la nation serbe. De ce fait, elle concurrence les autorités communistes serbes qui prétendent à elles seules représenter la population. Elle appelle les Serbes au retour à la foi en soulignant qu'un bon Serbe ne peut être qu'un orthodoxe pratiquant.

\section{L'ÉGLISE SERRBE ORTHODOXE DANS LES ANNÉES 1990 : ENTRE DEFENSE DE LA SERBITE ET RENOUVEAU MORAL}

Après la tenue des premières élections pluralistes en décembre 1990, l'Église serbe orthodoxe tend à s'émanciper du pouvoir, mais elle entretiendra avec lui des relations ambiguës tout au long des années 1990. Elle est confrontée à un régime qui a, certes, endossé le nationalisme comme ressource de légitimation mais qui demeure prudent face à certains acteurs nationalistes, se réclamant de valeurs plus traditionnelles. Si la messe de Noël est retransmise à la télévision en décembre 1990, le Parti socialiste serbe au pouvoir ne souhaite pas faire de Noël une fête nationale. En janvier 1991, le nouveau patriarche, Pavle, désigné en décembre 1990, adresse une lettre de protestation au Président,

\footnotetext{
28 Pravoslavlje, 01/07/88, p. 1.

29 Le prince Lazar reposa à Ravanica trois siècles avant que ses reliques ne fussent emportées lors de la grande migration du peuple serbe de 169 o. Elles finirent par aboutirent au monastère de Vrdnik où elles furent conservées pendant 245 ans. En 1942, sous l'occupation allemande, ses reliques furent transportées à Belgrade où elles demeurèrent jusqu'en juin 1988 .
}

30 Pravoslavlje, 15/10/88, p. 10. 
Slobodan Milošević, à ce propos. Lors des manifestations de l'opposition en mars 1991, l'Église ne manifeste pas son soutien au régime et prône la réconciliation. Pour elle, l'unité des Serbes est d'autant plus importante que les tensions s'aiguisent en Croatie entre Serbes et Croates. Au printemps 1991, l'Église serbe orthodoxe, ne contribuant pas à apaiser les esprits, poursuit sa politique d'inhumation des victimes des crimes des extrémistes ustaše pendant la Seconde Guerre mondiale. Au mois de mai 1991, le patriarche Pavle célèbre une messe à Jasenovac, lieu concentrationnaire où des dizaines de milliers de Serbes, Juifs et Tsiganes ont péris entre 1941 et 1945 (700 000 selon l'historiographie serbe dominante). L'organe de l'Église publie durant la même période une série de textes consacrés aux massacres contre les Serbes en Croatie pendant la Seconde Guerre mondiale.

L'Église serbe orthodoxe se place dès le début des incidents en Croatie aux côtés des Serbes de cette république. Elle agit auprès des acteurs politiques locaux (partis politiques d'opposition) et internationaux afin de défendre les intérêts des Serbes des " pays serbes occidentaux " (Croatie et BosnieHerzégovine). En octobre 1991, le patriarche Pavle s'adresse à Lord Carrington, président de la Conférence internationale sur la Yougoslavie, déclarant que les Serbes ne peuvent plus vivre aux côtés des Croates étant donné le génocide perpétré à leur encontre pendant la Seconde Guerre mondiale. Il préconise que les Serbes de Croatie vivent sous le même toit étatique que les autres Serbes. En janvier 1992, lors du synode des évêques de l'Église serbe orthodoxe, un message de soutien est prononcé en faveur de l'autonomie politique des Serbes de Bosnie-Herzégovine. L'Église souhaite s'imposer comme interlocutrice et représentante de l'ensemble des Serbes d'ex-Yougoslavie, et conteste au pouvoir de Belgrade le droit de parler au nom de tous les Serbes. Elle veut se poser en principal arbitre moral de la vie politique en Serbie.

Dans le contexte de la désintégration de la Yougoslavie communiste, l'Église serbe orthodoxe ne limite pas son action aux frontières de la République de Serbie ou de la République fédérale de Yougoslavie instituée au printemps 1992. En effet, les éparchies composant l'Église serbe orthodoxe concernent de nombreux territoires situés en dehors des limites de la Serbie. Son territoire de référence n'est donc pas celui de l'État de Serbie, mais celui où sont établis ses éparchies et diocèses (en dehors de ceux constitués dans le monde occidental). L'ensemble de ces territoires constitue la " terre sacrée serbe ", amenée à être intégrée dans un seul cadre étatique.

Si l'Église ne diffuse pas à proprement parler un discours de haine à l'encontre des Croates catholiques et des Bosniaques musulmans, elle nie que les Serbes soient responsables de l'éclatement du conflit. À la dénonciation des crimes perpétrés par les forces armées serbes, les ecclésiastiques opposent les crimes commis contre les Serbes de Croatie et de Bosnie-Herzégovine. Ils récusent l'accusation selon laquelle les Serbes sont les agresseurs en arguant qu'ils 
sont autochtones en Bosnie-Herzégovine et que par conséquent ils ne peuvent pas être considérés comme des occupants. Selon l'Église serbe orthodoxe, les Serbes ont été victimes d'un " génocide spirituel ", de nombreux lieux de culte ayant été volontairement détruits par leurs ennemis ${ }^{31}$. Ils estiment que tous les Serbes doivent vivre sous un seul et même toit étatique. Selon eux, les frontières entre les républiques yougoslaves ont été imposées par des " révolutionnaires marxistes " et ne sauraient être acceptées ${ }^{32}$.

Au cours des années de guerre, l'Église serbe orthodoxe se sent plus proche du pouvoir politique serbe de Bosnie-Herzégovine que de Serbie. En effet, les autorités de Pale, sous la direction de Radovan Karadžić, soutiennent les valeurs traditionnelles de la société serbe, à savoir la monarchie et la religion orthodoxe. Alors que le catéchisme a été introduit dans le système scolaire de la Republika Srpska, les autorités de Belgrade refusent qu'il soit enseigné. L'Église n'aura de cesse de réclamer au cours des années 1990 l'introduction du catéchisme dans les écoles. Lors du conflit politique opposant entre 1993 et 1995 les dirigeants serbes de Bosnie-Herzégovine et de Serbie, l'Église se place du côté de la Republika Srpska et dénonce l'embargo imposé par Belgrade en 1994 à l'entité serbe de Bosnie-Herzégovine ${ }^{33}$. Néanmoins, le patriarche Pavle ne se déclare pas hostile à l'idée que Slobodan Milošević représente les intérêts serbes aux négociations de Dayton au nom des Serbes de Bosnie-Herzégovine. À la fin du conflit en Bosnie-Herzégovine, elle dénonce les « injustices " des accords de paix de Dayton, selon elle, défavorables aux Serbes. En 1996, elle manifeste son inquiétude face à la justice internationale et plus particulièrement au TPIY (Tribunal Pénal International pour l'ex-Yougoslavie) de La Haye jugeant, selon elle, uniquement les dirigeants serbes et, à travers eux, le peuple serbe 34 .

En même temps que l'Église serbe orthodoxe s'investit dans la défense des intérêts des Serbes de Croatie et de Bosnie-Herzégovine, elle œuvre au renouveau moral et spirituel du peuple serbe en Serbie même. Elle déplore que l'éducation nationale soit sous l'emprise du marxisme. Elle critique l'esprit consumériste, l'influence néfaste des sectes qui, selon elle, menace de détruire la foi chrétienne. Au cours des années 1990, elle se distancie du pouvoir, considéré comme communiste, et lors du grand mouvement de contestation civique de l'hiver 1996-1997, elle dénonce avec fermeté la falsification des résultats électoraux, tout en rappelant la trahison par le pouvoir des " pays serbes occidentaux $» 35$.

${ }^{1}$ Pravoslavlje, (697), 01/04/96, p. 1.

32 Pravoslavlje, (664), 01-14/11/94, p. 2.

33 Pravoslavlje, (664), 01-14/11/94, p. 1.

34 Pravoslavlje, (701), 01/06/1996, p.1.

35 Pravoslavlje, (716), 15/o1/97, p. 1. 


\section{L'ÉGLISE SERBE ORTHODOXE APRÈS LA CHUTE DU REGIME DE SLOBODAN MILOŠEVIĆ}

Depuis la chute du régime de Slobodan Milošević en octobre 2000, les relations entre le pouvoir politique et l'Église serbe orthodoxe se sont largement améliorées. En effet, l'Église s'était montrée très critique à l'égard du régime de Slobodan Milošević qui gardait une dimension laïque et, selon elle, marxiste. Elle était davantage proche d'une grande partie de l'opposition démocratique de Serbie qui tenait un discours anticommuniste.

En juillet 2001, l'État a cédé à la demande de l'Église serbe orthodoxe d'introduire le catéchisme dans le système scolaire de Serbie. Cette mesure a été interprétée comme une concession du gouvernement de Zoran Djindjić à la suite du transfert à La Haye de l'ancien Président yougoslave, Slobodan Milošević. En effet, cette mesure a été prise dans la précipitation, sans réflexion en amont, sans réelle concertation avec le Patriarcat et surtout à l'encontre de la Constitution stipulant la séparation de l'État et des Églises ${ }^{36}$. L'Église orthodoxe agit en direction de la jeunesse et considère que l'on ne peut la séparer de l'école, comme la mère d'un enfant. Le dogme religieux orthodoxe doit être le fondement de l'éducation morale et tous ceux qui s'y opposent sont considérés comme des " disciples de Satan $" 37$.

Néanmoins, l'État n'a pas accédé à l'ensemble des demandes de l'Église serbe orthodoxe qui souhaite, en particulier, que l'orthodoxie devienne une religion d'État supérieure aux autres religions sur un plan juridique. L'Église serbe rejette le principe de sécularisation et est soutenue en cela par une partie de l'élite politique, en particulier par le Parti démocratique de Serbie du Premier ministre Vojislav Koštunica ${ }^{38}$.

En canonisant l'évêque d'Ohrid et de Ziča, Nikolaj Velimirović (1880-1956), le 24 mai 2003 , le synode de l'Église serbe orthodoxe a franchi la dernière étape du processus de réhabilitation de l'ecclésiastique controversé, qui avait débuté au cours des années 1980 et était porté par le courant autour d'Amfilohije Radović et Atanasije Jevtić. Désormais, cet évêque a pris une place significative dans l'histoire de l'Église, peut-être plus importante que celle de Saint Sava, fondateur de l'Église serbe orthodoxe en 1219. Même si l'Église serbe orthodoxe soutient officiellement la démocratisation de la société serbe, cette canonisa-

\footnotetext{
${ }^{6}$ Popović-Obradović (Olga), "Crkva, nacija, država ija u Srbiji ” (Église, nation, État : l’Église serbe orthodoxe et la transition en Serbie), in Izmedju autoritarizma i demokratije : Srbija, Crna Gora, Hrvatska, knjiga II, Civilno društvo i politička kultura (Entre autoritarisme et démocratieonténégro, Croatie, livre II, La société civile et la culture politique), Beograd : CEDET, 2004, pp. 137-138.

37 Selon un communiqué du service d'information de l'Église serbe orthodoxe du 24/11/oo.

$3^{8}$ À titre d'illustration, voir le message adressé par Vojislav Koštunica à l'occasion d'un colloque organisé le 4 janvier 2003 sur l'évêque Nikolaj Velimirović. Politika, 05/01/03, p. 7.
} 
tion l'inscrit paradoxalement dans un courant de pensée orientée contre l'Europe, le monde occidental en général et la démocratie 39 .

Malgré le retour de la paix en Croatie, Bosnie-Herzégovine et au Kosovo, l'Église serbe orthodoxe est impliquée dans des questions politiques et territoriales. L'avenir du Kosovo est au cœur de ses préoccupations, tant le patrimoine de l'Église y est riche et son identité liée aux événements historiques qui se sont produits dans cette région. Selon le métropolite du Monténégro et du littoral, Amfilohije Radović, une bataille a été perdue (par un incroyant : Slobodan Milošević), mais l'Église et le peuple serbe ne peuvent être vaincus. Perdre le Kosovo reviendrait à " être rayé de l'histoire et de l'humanité "40. L'Église serbe orthodoxe est contestée au Monténégro où une Église monténégrine orthodoxe s'est constituée en octobre 1993. Au Monténégro, il y a deux façons d'être orthodoxe qui dépendent de la nation à laquelle on appartient : monténégrine ou serbe. Par ailleurs, l'Église serbe orthodoxe conteste l'autocéphalie de l'Église macédonienne orthodoxe prononcée en 1967 mais non reconnue sur le plan canonique. En 2002, l'Église serbe lui a proposé un accord pour sortir de la situation bloquée depuis la fin des années 1960 : elle se déclare prête à reconnaître l'autonomie de l'Église macédonienne à condition que celle-ci renonce à l'autocéphalie. Cette offre a été rejetée par le chef de l'Église macédonienne orthodoxe ${ }^{41}$. L'Église serbe orthodoxe a provoqué de nouvelles tensions entre les deux Églises : elle a renforcé le statut de sa branche en Macédoine en l'élevant au rang d'archevêché (d'Ohrid) auquel une autonomie a été reconnue au mois de mai 2005.

\section{RENOUVEAU DE LA FOI ?}

En l'espace d'une vingtaine d'années, la religion orthodoxe est redevenue une religion de référence pour les habitants de Serbie et un élément important de leur identité. Alors que, sous le régime communiste, la norme était de se déclarer athée (ce qui ne signifie pas que toutes les personnes l'étaient), de nos jours, elle est de s'affirmer orthodoxe. Pour autant, le nombre de pratiquants n'a pas augmenté de manière significative. Selon le dernier recensement de la population de 2002, 6371584 personnes ont déclaré qu'elles appartenaient à la confession orthodoxe, soit 84,98\% de la population totale de Serbie (7 498001

39 Ce courant antidémocratique est représenté par les évêques renommés Atanasije Jevtić, Amfilohije Radović, Danilo Krstić et Irinej Bulović. Danas, 31/05/05, p. 4.

$4 \circ$ Entretien du métropolite, Amfilohije Radović, au quotidien Blic, 13/12/99.

${ }^{11}$ Popović-Obradović (Olga), art.cit., pp. 145-146. In faut noter que le Métropolite Jovan de Veles a accepté l'invitation de l'Église serbe orthodoxe. Il a été exclu de l'Église macédonienne orthodoxe. 
habitants). Néanmoins cette indication ne nous renseigne pas réellement sur le degré de religiosité des habitants concernés. En revanche, peu de personnes ont précisé qu'elles n'étaient pas croyantes mais athées : 40068 , soit $0.53 \%$ de la population totale ${ }^{42}$. On a donc assisté au début des années 1990 à un retournement de situation et ce phénomène n'a fait que se renforcer depuis.

Une enquête réalisée en 2004 indiquait que $84,9 \%$ de la population serbe majeure était croyante et que $89,4 \%$ était baptisée. Néanmoins, seulement 17,2 $\%$ de ces personnes déclaraient aller régulièrement à l'église, $17,6 \%$ souvent et $23,4 \%$ rarement 43 . Les études sociologiques réalisées au cours des années 1990 ont mis en relief un retour de la population serbe à la foi orthodoxe. Le degré de religiosité a évolué au cours de la dernière décennie : c'est en 1993-1994 qu'il a été le plus élevé, au moment où le pays sombrait dans l'hyperinflation et cherchait à s'extirper du conflit armé en Bosnie-Herzégovine. Le taux de religiosité de la population s'établissait à $60 \%$ environ en $1999^{44}$.

Néanmoins, le fait de se déclarer croyant et pratiquant ne nous renseigne pas en profondeur sur la religiosité. Nombre d'enquêtes sociologiques révèlent un écart entre le sentiment d'appartenance à l'orthodoxie et la religiosité. Si 70 $\%$ des citoyens de Serbie fêtent Noël par conviction et tradition, seuls $20 \%$ déclarent croire réellement en Dieu tandis que $41 \%$ affirment ne pas croire en lui45. Une bonne partie de la population doute de sa religiosité. En outre, les citoyens de Serbie ne lient pas la religiosité à la fréquentation régulière de l'église. On enregistre un degré de religiosité différent en fonction des générations : les plus religieux étant les personnes les plus âgées et les plus jeunes tandis que la génération des 40-50 ans l'est moins.

Il est intéressant de noter que les athées autrefois très nombreux sont désormais une portion congrue de la population et qu'un rejet se manifeste à l'égard de ces personnes puisque, selon une étude de l'UNDP, $30 \%$ des citoyens de Serbie ne souhaiteraient pas qu'un de leurs proches se marie avec un ou une athée ${ }^{46}$.

42 Soit quatre fois moins qu'en 1991 (160 o00 personnes). " Povratak tradiciji n, Politika, 31/05/03. p. 1.

43 Radio Bg2, 13/04/O4.

44 Politika, 31/05/03.

45 Selon une enquête réalisée en 2003. "Božić se poštuje i slavi u Srbiji ", Politika, 06/01/2003.

46 Izveštaj o humanom razvoju. Srbija 2005 : snaga različitosti (Rapport sur le développement humain. Serbie 2005 : la force de la différence), Beograd : UNDP, 2005. 


\section{CONCLUSION}

L'Église serbe orthodoxe est depuis une quinzaine d'années une des premières institutions dans laquelle les citoyens serbes ont confiance. En décembre $2004,71 \%$ des citoyens de Serbie déclaraient avoir confiance dans l'Église contre $51 \%$ pour le Président de la République et $33 \%$ pour le gouvernement de Serbie 47 .

Dans un monde en pleine mutation, l'Église serbe orthodoxe apparait comme une source de stabilité et de continuité. Néanmoins, si l'Église s'impose comme un acteur social significatif en Serbie, c'est moins en diffusant une culture religieuse qu'en défendant les intérêts nationaux serbes. Avant d'ètre orthodoxe, l'Église est serbe, tout comme l'indique le nom officiel de cette structure. L'Église serbe orthodoxe est sortie de la marginalité au cours des années 1980, en particulier au moment de la grande vague nationaliste des années 1987-1989. Elle s'est impliquée largement dans la défense des Serbes de Croatie et de Bosnie-Herzégovine pendant les conflits armés des années 1990 en soutenant les différentes directions politiques qu'ils s'étaient données. Sa plus grande visibilité dans l'espace social et son action n'ont pas pour autant contribué à un renforcement significatif de la religiosité des citoyens serbes, même si ceux-ci marquent leur attachement à l'orthodoxie en tant qu'élément constitutif de l'identité nationale serbe.

47 Poverenje u institucije i spremnost gradjana na politicku participaciju (Confiance dans les institutions et disposition des citoyens à participer à la vie politique), Beograd : Centar za politikološka istrażivanja i javno mnenje, 2005. Document en ligne : 〈http://www.cpijm.org.yu/scharts/Izvestaj3.pdf $>$ (consulté le 27 septembre 2005). 